\title{
Cellular Uptake and Efflux of Palbociclib In Vitro in Single Cell and Spheroid Models $\$$
}

\author{
M. Jove, ${ }^{1}$ J.A. Spencer, ${ }^{1}$ M.E. Hubbard, E.C. Holden, R.D. O’Dea, B.S. Brook, R.M. Phillips, \\ S.W. Smye, P.M. Loadman, and C.J. Twelves
}

Institut Català d'Oncologia, Medical Oncology Department, Barcelona, Spain (M.J.); Institute of Cancer Therapeutics, University of Bradford, Bradford, United Kingdom (J.A.S., P.M.L.); School of Mathematical Sciences, University of Nottingham, University Park, Nottingham, United Kingdom (M.E.H., E.C.H., R.D.O., B.S.B.); School of Applied Sciences, University of Huddersfield, Queensgate, Huddersfield, United Kingdom (R.M.P.); School of Medicine, University of Leeds, Leeds, United Kingdom (S.W.S.); and University of Leeds and Leeds Teaching Hospitals, National Health Service Trust, St. James's University Hospital, Leeds, United Kingdom (C.J.T.)

Received February 13, 2019; accepted June 6, 2019

\begin{abstract}
Adequate drug distribution through tumors is essential for treatment to be effective. Palbociclib is a cyclin-dependent kinase 4/6 inhibitor approved for use in patients with hormone receptor positive, human epidermal growth factor receptor 2 negative metastatic breast cancer. It has unusual physicochemical properties, which may significantly influence its distribution in tumor tissue. We studied the penetration and distribution of palbociclib in vitro, including the use of multicellular threedimensional models and mathematical modeling. MCF-7 and DLD-1 cell lines were grown as single cell suspensions (SCS) and spheroids; palbociclib uptake and efflux were studied using liquid chromatography-tandem mass spectrometry. Intracellular concentrations of palbociclib for MCF-7 SCS $\left(C_{\max } 3.22 \mu \mathrm{M}\right)$ and spheroids $\left(C_{\max } 2.91 \mu \mathrm{M}\right)$ were 32 - and 29 -fold higher and in DLD-1, 13- and 7-fold higher, respectively, than the media concentration $(0.1 \mu \mathrm{M})$. Total palbociclib uptake was lower in DLD-1 cells than MCF-7 cells in both SCS and spheroids. Both uptake and efflux of palbociclib were slower in spheroids than SCS. These data were used to develop a mathematical model of

palbociclib transport that quantifies key parameters determining drug penetration and distribution. The model reproduced qualitatively most features of the experimental data and distinguished between SCS and spheroids, providing additional support for hypotheses derived from the experimental data. Mathematical modeling has the potential for translating in vitro data into clinically relevant estimates of tumor drug concentrations.

\section{SIGNIFICANCE STATEMENT}

This study explores palbociclib uptake and efflux in single cell suspension and spheroid models of cancer. Large intracellular concentrations of palbociclib are found after drug exposure. The data from this study may aid understanding of the intratumoural pharmacokinetics of palbociclib, which is useful in understanding how drug distributes within tumor tissue and optimizing drug efficacy. Biomathematical modelling has the potential to derive intratumoural drug concentrations from plasma pharmacokinetics in patients.
\end{abstract}

\section{Introduction}

For cancer therapeutics to be effective, drug concentrations must exceed a threshold level within cancer cells for a period of time sufficient to ensure the desired effect. Pharmacokinetic resistance, the ineffective delivery of the drug to the cell, has been recognized as an important contributor to treatment failure (Minchinton and Tannock, 2006). The tumor microstructure plays an important role in pharmacokinetic resistance, because cancer drugs have to penetrate and distribute throughout the tumor to be optimally effective

Funding for this research was provided from a Grant for Translational Research and a grant from Leeds National Health Service Charitable Trustees.

${ }^{1}$ M.J. and J.A.S. contributed equally to this work

https://doi.org/10.1124/jpet.119.256693.

S This article has supplemental material available at jpet.aspetjournals.org.
(Minchinton and Tannock, 2006). The physicochemical properties of each drug also influence its delivery and distribution; the molecular weight, polarity, $\mathrm{pKa}$, and extent of protein binding of a drug all affect how it distributes within the tumor (Fuso Nerini et al., 2014).

Most preclinical intratumoral pharmacokinetic (PK) data relate to cytotoxicity testing in vitro, using measures such as $\mathrm{IC}_{50}$ and plasma $\mathrm{PK}$ in vivo; the majority of preclinical drug distribution studies focus on distribution within individual organs rather than tumors. Although there is a trend to investigate drug penetration using in vitro tissue models in preclinical drug development (Katt et al., 2016), these studies are not usually routinely performed. In vitro multicellular models using cancer cells are feasible and potentially useful to answer questions around drug penetration and distribution at a tissue or cellular level (Evans et al., 2009; Barrera-Rodríguez

ABBREVIATIONS: Ab, antibody; AUC, area under the concentration-time curve; CDK, cyclin-dependent kinase; CV, coefficient of variation; 5 FU, 5-fluorouracil; LC-MS/MS, liquid chromatography-tandem mass spectrometry; MP, mobile phase; PK, pharmacokinetic; pRb, phosphoretinoblastoma; Rb, retinoblastoma; SCS, single cells in suspension. 
and Fuentes, 2015; Winter et al., 2019). Multicellular models have been used previously to study intratumoral drug penetration (Godugu et al., 2013; Huang and Gao, 2018). Moreover, some of the PK parameters derived from such models can be used to develop biomathematical models that can improve the understanding of key factors that govern intratumoral drug delivery (Evans et al., 2009; Groh et al., 2014). These models are limited by the parameters obtained from experiments, but can potentially optimize the drug development process by identifying promising drug combinations and schedules, supplementing in vivo studies that are resource intensive and involve ethical issues and so are limited in number (Altrock et al., 2015).

Once a drug enters clinical trials, plasma PK studies are routine, but there are few drugs for which intratumoral drug concentrations and/or distribution have been studied. One exception is the fluoropyrimidine prodrug capecitabine, where a PK study confirmed preferential conversion to 5-fluorouracil (5FU) in the tumor (Schüller et al., 2000).

Palbociclib (Ibrance; Pfizer) is an oral cyclin-dependent kinase (CDK) 4/6 inhibitor that blocks phosphorylation of the retinoblastoma $(\mathrm{Rb})$ protein, a tumor suppressor that controls the transition from G1 to $\mathrm{S}$ phase of the cell cycle. Palbociclib and other CDK4/6 inhibitors are approved in combination with endocrine therapy in patients with hormone receptor-positive, human epidermal growth factor receptor 2 negative metastatic breast cancer (www.ema.europa.eu; 2015), in whom the combination approximately doubles progression-free survival (Clark et al., 2016). Palbociclib has unusual physicochemical properties, which are likely to have a significant influence on its penetration and distribution through tissues. Namely, it has a very large volume of distribution of 2583 liters and a half-life of 26 hours, indicating extensive drug binding to peripheral tissues (Schwartz et al., 2011; Flaherty et al., 2012). Furthermore, in whole blood, palbociclib distributes preferentially into the cellular component over plasma, with a whole blood-to-plasma concentration ratio of 1.63 (www.ema.europa.eu). Separate studies have reported plasma palbociclib PK (Smith et al., 2011) and intratumoural palbociclib concentrations in vivo (Nguyen et al., 2010) but were based on different doses and routes of administration. Reported clinical plasma concentrations for palbociclib range from 0.062 to $0.278 \mu \mathrm{M}$ (https://www.accessdata.fda. gov/; Tamura et al., 2016; Masuda et al., 2018; Taylor et al., 2018). Studies have not reported a direct comparison of both intratumoral and plasma palbociclib concentrations, except in the case of glioblastoma, where the issue is complicated by the blood-brain barrier (Taylor et al., 2018).

Better understanding of intratumoral palbociclib PK may inform the design of new clinical trials. For example, in studies combining cell cycle inhibitors with chemotherapy, cell cycle arrest may reduce the efficacy of chemotherapy, leading to antagonistic rather than synergistic effects (Dean et al., 2012; McClendon et al., 2012); alternative dosing or scheduling may overcome such a limitation.

We have developed a methodology to study drug uptake and efflux in SCS and 3D spheroid models, quantifying drug concentrations using liquid chromatography-tandem mass spectrometry (LC-MS/MS). This allows us to explore the ability of the drug to move through single cells and spheroids. Using these data, we developed a mathematical model of palbociclib transport through cancer cells that can quantify the role of key parameters, which determine drug penetration and distribution within the tumor.

\section{Materials and Methods}

\section{LC-MS/MS Methodology}

Stock solutions of palbociclib were prepared at $1 \mathrm{mM}$ in DMSO and stored at $-20^{\circ} \mathrm{C}$. Standard solutions for system calibration were made by further dilutions of stock solutions with a mixture of $50 \%$ mobile phase A (MPA) and 50\% mobile phase B (MPB). For each set of experiments run on LC-MS/MS, calibration curves were created between 0.02 and $10 \mu \mathrm{M}$.

Samples were separated and analyzed on a Waters 2695 highperformance liquid chromatography system in combination with a Waters 2996 Diode Array Detector and a Waters Micromass Ultima triple quadrupole MS. Samples were maintained at $4^{\circ} \mathrm{C}$ in the autosampler and $10 \mu \mathrm{l}$ was injected for analysis. Sample separation was carried out on a HiChrom RPB column $(250 \times 2.1 \mathrm{~mm}$ internal diameter, $5 \mu \mathrm{m}$ particle size) maintained at $40^{\circ} \mathrm{C}$. Gradient elution was applied with $10 \%$ acetonitrile and $0.1 \%$ formic acid for MPA and $50 \%$ acetonitrile and $0.1 \%$ formic acid for mobile phase B (MPB) with the following gradient: the starting eluent consisted of 70\% MPA and $30 \% \mathrm{MPB}$, the proportion of MPB increasing linearly to $80 \%$ over 5 minutes and then returning to $30 \%$ over 1 minute. The column was re-equilibrated at starting conditions for 4 minutes. The flow rate was $0.5 \mathrm{ml} \cdot \mathrm{min}^{-1}$ and split post-column with $0.3 \mathrm{ml} \cdot \mathrm{min}^{-1}$ delivered to the mass spectrometer. Palbociclib was detected by monitoring the mass transition $\mathrm{m} / z 448.73>381.04$ with the instrument operating in positive mode. A cone energy of $20 \mathrm{eV}$ and collision energy of $20 \mathrm{eV}$ was used with argon gas as the collision gas. The UV signal of palbociclib was also monitored at a wavelength of $365 \mathrm{~nm}$.

\section{Cell Culture}

The human breast cancer cell line MCF-7 and the human colorectal cancer cell line DLD-1 were obtained from our laboratory. Cell line provenance was verified by annual STR profiling (European Collection of Authenticated Cell Cultures, Public Health England, Porton Down, Salisbury, UK), and bimonthly mycoplasma testing was regularly undertaken in house using a MycoProbe Mycoplasma Detection Kit (R\&D Systems, Inc., Minneapolis, MN). Both cell lines have the capability to form spheroids.

Cells were cultured in Dulbecco's modified Eagle's medium high glucose, supplemented with $10 \%$ fetal bovine serum and 5\% L-glutamine. For MCF-7 cell line, 1\% insulin was also added. Spheroids were created using 96 -well plates previously coated with $1 \%$ Agarose. We optimized spheroid formation to achieve a diameter of approximately $400 \mu \mathrm{m}$ on day 4 (collecting day) by seeding at $3500 \mathrm{cells} \cdot \mathrm{ml}^{-1}$ for MCF-7 and 6000 cells.ml ${ }^{-1}$ for DLD-1. Spheroid diameter was optimized to avoid the formation of a necrotic core and reduce variability in the subsequent assays. Briefly, spheroids were embedded in paraffin, sectioned, and stained with hematoxylin-eosin to visualize their structure and confirm the absence of a necrotic core (Supplemental Fig. S3). Cells were maintained in $25 \mathrm{ml}$ tissue culture flasks at $37^{\circ} \mathrm{C}$ in humidified incubator with $5 \% \mathrm{CO}_{2}$.

\section{Cell Viability}

Both MCF-7 and DLD-1 were treated for 480 minutes with $0.1 \mu \mathrm{M}$ of palbociclib under the conditions described below for both uptake and efflux experiments, with viable cells counted using a hemocytometer and trypan blue.

\section{Western Blots}

We performed Western blots of retinoblastoma $(\mathrm{Rb})$, phosphoretinoblastoma ( $\mathrm{pRb}$; serine 780), CDK4, and CDK6 as a pharmacodynamics measure of whether palbociclib' s mechanism of action was preserved in the cell lines. 
MCF-7 and DLD-1 cells were grown as described above and, once confluent, harvested for analysis. Cell pellets were lysed for 10 minutes on ice in buffer containing a protease and phosphatase inhibitor cocktail mix (Abcam), followed by sonication using $3 \times 5$ second cycles twice at $40 \%$ power. Samples were tested for protein content by Bradford assay and loaded on to 10\% SDS-polyacrylamide gels at $50 \mu \mathrm{g} \cdot \mathrm{well}^{-1}$ protein for separation. A protein ladder was also loaded for molecular weight comparison (PageRuler Plus Prestained Protein Ladder, Fermentas Life Sciences). Separated proteins were then transferred onto a nitrocellulose membrane and incubated with blocking buffer appropriate to the $1^{0}$ antibody (Ab) for 1 hour. Membranes were subsequently incubated with the following $1^{\circ} \mathrm{Abs}$ overnight at $4^{\circ} \mathrm{C}$ : CDK4 (D9G3E) rabbit mAb, CDK6 (DCS83) mouse mAb, Rb (4H1) mouse mAb, phospho-Rb (Ser780) (D59B7) rabbit mAb, phospho-Rb (Ser795) Ab and phospho-Rb (Ser807/811) (D20B12) XP rabbit mAb. $\beta$-Actin $\mathrm{Ab}$ was used as a control. Following incubation with the appropriate $2^{\circ} \mathrm{Ab}$ for 1 hour at room temperature, the membranes were incubated with SignalFire ECL Reagent (Cell Signaling Technology, Inc., Danvers, MA) for 1 minute and the signal captured on $\mathrm{X}$-ray film. Exposure time varied from 10 seconds to 3 minutes, depending on the antibody used.

\section{In Vitro Drug Uptake and Efflux}

The palbociclib concentration used for uptake and efflux experiments was $0.1 \mu \mathrm{M}$. This dose was selected based on reported clinical plasma concentrations (https://www.accessdata.fda.gov/; Tamura et al., 2016; Masuda et al., 2018; Taylor et al., 2018). A concentration at the lower end of the range was chosen to assure cell viability while remaining above the biologically effective dose of $0.06 \mu \mathrm{M}$ (https:// www.accessdata.fda.gov/).

Drug Uptake. One million cells in suspension, or 30 spheroids per time point, were maintained at $37^{\circ} \mathrm{C}$ in Eppendorf tubes and treated with $1 \mathrm{ml}$ of $0.1 \mu \mathrm{M}$ palbociclib in complete media for $0-240(0,5,10$, $20,40,60,120$, and 240 minute) and $0-480$ minutes $(0,5,10,20,40,60$, $120,240,360$, and 480 minutes), respectively. At each designated time point, cells were centrifuged at $1000 \mathrm{~g}$ for 2 minutes, media were discarded, and the cell pellet or spheroids were washed three times with PBS before resuspension in $150 \mu \mathrm{l}$ of acetonitrile and storing on ice. The cell pellet or spheroids were sonicated twice at $40 \%$ power for $3 \times 5$-second cycles and $6 \times 5$-second cycles, respectively, using a Sonoplus HD2070 Homogenizer Ultrasonic (Scientific Laboratory Supplies Limited, Nottingham, UK) to disrupt the cell membranes and release intracellular drug. All samples were then centrifuged at $10,000 \mathrm{~g}$ for 5 minutes. Finally the supernatant was transferred to a new Eppendorf tube and evaporated using a Genevac personal evaporator at $37^{\circ} \mathrm{C}, 8$ mbar pressure for approximately 120 minutes and reconstituted in $30 \mu \mathrm{l}$ of mobile phase (MPA:MPB, 1:1). Appropriate control samples of untreated cells or spheroids and samples containing drug without cells were also included to ensure the validity of the assay. The method is described in Supplemental Fig. S1.

Drug Efflux. One million cells in suspension or 30 spheroids were treated with $1 \mathrm{ml}$ of $0.1 \mu \mathrm{M}$ palbociclib in complete media for 1 and 2 hours, respectively, at $37^{\circ} \mathrm{C}$. After incubation with drug, samples were centrifuged at $1000 \mathrm{~g}$ for 2 minutes, media containing drug removed, and the cell pellet or spheroids washed three times with PBS. Fifty microliters of fresh drug-free media was then added and samples were incubated for 5-240 (5, 10, 20, 40, 60, 120, and 240 minutes) and 5-480 minutes (5, 10, 20, 40, 60, 120, 240, 360, and 480 minutes) for cells and spheroids, respectively. At each designated time point, samples were centrifuged for 2 minutes at $1000 \mathrm{~g}$ and the media transferred to a clean Eppendorf tube. Both media and cell or spheroid pellets were processed for analysis as described above. The method is described in Supplemental Fig. S2.

Protein concentration for each experiment was measured using the Bradford assay. Cell and spheroid volumes were derived from their respective diameters assuming both to be spherical. Diameters were measured accurately using a metric rule under the microscope at $\times 20$ and validated by Image $J$ software for scientific analysis. For single cell diameters, at least 50 cell diameters were measured in different passages; the mean cell diameter was used to derive the cell volume. For spheroids, the diameter of 15 spheroids was measured in each experiment and the mean diameter used to calculate the spheroid volume. Each experiment was performed in triplicate.

\section{Data Analysis}

SCS and spheroid pellets were diluted at the end of the experiment in $30 \mu \mathrm{l}$ MPA. To determine the actual concentration of drug in the cell or spheroid pellets, the following equation applied (all volumes in microliters):

SCS.

$$
\begin{aligned}
\text { Dilution factor }= & {\left[\left(\text { volume of a single cell } \times 1 \times 10^{6} \text { cells }\right)\right.} \\
& +30 \mu \mathrm{l}] / \text { volume of a single cell } \times 1 \times 10^{6} \text { cells }
\end{aligned}
$$

\section{Spheroids.}

$$
\begin{aligned}
\text { Dilution factor }= & {[(\text { volume of a spheroid } \times 30 \text { spheroids })} \\
& +30 \mu l] / \text { volume of a spheroid } \times 30 \text { spheroids. }
\end{aligned}
$$

We also expressed drug concentrations relative to the sample protein concentration for each experiment (drug concentration:protein ratio), which is independent of cell or spheroid volume.

Raw LC-MS data were processed in MassLynx V4.1. and graphs plotted in Graph Pad PRISM 6.

\section{Statistics}

Statistical comparison of PK parameters [e.g., area under the concentration-time curve (AUC), total drug uptake/efflux, maximum concentration $\left(C_{\max }\right)$, time at which maximum concentration is observed, and rate of uptake/efflux) between cells and spheroids were made using Graph Pad PRISM 6. Both $C_{\max }$ and time at which maximum concentration is observed were derived from direct observation of GraphPad PK curves. All graphs were represented with mean plus error bars with three independent data points per time point; therefore we consider that representing descriptive data were more appropriate. Experiments were performed in triplicate, which precludes the assumption of a normal distribution of the data; the Mann-Whitney $U$ test was, therefore, used for statistical comparisons. We used coefficient of variation (CV) to determine the technical error of drug uptake and efflux experiments. Technical error was tested performing three repeats in SCS at the 60-minute drug uptake time point, with a CV of $19.4 \%$, and in SCS at the 6-hour drug efflux time point, with a $\mathrm{CV}$ of $32.5 \%$.

\section{Mathematical Modeling}

The mathematical model used to describe the transport of palbociclib in single cells and in spheroids has been described previously (Hubbard et al., 2017). In summary, the interaction of palbociclib with the microenvironment of cells is restricted to drug binding and described by a three-compartment model, comprising extracellular space (volume $\mathrm{V}_{1}, 1 \mathrm{ml}$ for uptake experiments, $50 \mu \mathrm{l}$ for efflux experiments) with a uniform concentration $\mathrm{C}_{1}$ of free drug, and intracellular space (volume $\mathrm{V}_{2}, 1.65 \mu \mathrm{l}$ for DLD-1 and $3.28 \mu \mathrm{l}$ for MCF-7), with concentrations $\mathrm{C}_{2}$ and $\mathrm{C}_{3}$ corresponding to free and bound drug, respectively. The term "bound" refers to all types of binding taking place within the cell, including to protein, lipids, and organelles. It is assumed that binding can be described by a simple kinetic model with drug binding reversibly to sites within the cell. The core model equations are given by: 


$$
\begin{aligned}
& V_{1} \frac{d C_{1}}{d t}=a k_{1}\left(C_{2}-C_{1}\right), \\
& V_{2} \frac{d C_{2}}{d t}=a k_{1}\left(C_{1}-C_{2}\right)-V_{2} k_{2} C_{2}\left(C_{0}-C_{3}\right)+V_{2} k_{-2} C_{3}, \\
& V_{2} \frac{d C_{3}}{d t}=V_{2} k_{2} C_{2}\left(C_{0}-C_{3}\right)-V_{2} k_{-2} C_{3},
\end{aligned}
$$

where $a$ is the total cellular surface area $\left(6.75 \times 10^{-4} \mathrm{~m}^{2}\right.$ for DLD-1, $1.07 \times 10^{-3} \mathrm{~m}^{2}$ for MCF-7 cells). These equations contain four parameters that are found using a least-squares fit to the experimental data-these are $k_{1}$, the rate of transport across the cell membrane; $k_{2}$, the binding rate; $k_{-2}$, the unbinding rate; and $C_{0}$, the concentration of binding sites in the cells.

The model adopts a compartmental approach in which it is assumed that there is a rapid equilibration of concentration within the extracellular volume and that, for SCS, the free and bound fractions of drug are evenly distributed within the intracellular compartment. For spheroid modeling we no longer assume that equilibration of drug throughout the spheroid is instantaneous, but instead model the spatial variation of drug concentration within the spheroid using diffusive transport. The spheroid model is derived by means of a multiscale homogenization technique developed previously (Sanchez-Palencia, 1970; Larsen, 1975; Babuška, 1976) and recently applied to relevant biologic contexts (Shipley and Chapman, 2010; (Dalwadi et al., 2018)). Full details of the analysis (Supplemental Materials 2) are described by Holden (2018). In brief, this allows for the derivation of a macroscale description of the drug dynamics, based on the kinetic model, which accommodates microscale features of drug transport and binding kinetics.

The spheroid model introduces an additional parameter that represents a diffusion rate, the value of which is found with the other four parameter values using a least-squares fit to the spheroid data (Supplemental Materials 1). The numerical model divides the spheroid into a series of concentric layers, and it is assumed that the dynamics of drug transport can be attributed to the exchange of free drug between these layers in the spheroids and between the extra- and intracellular compartments, and binding/unbinding within the intracellular compartment. In line with the experimental procedure described above, the parameter-fitting procedure is undertaken for modeling the uptake of drug by initially drug-free cells and for modeling the efflux of drug from cells initially bathed in drug. The model parameters are estimated for each cell line by fitting to all six available datasets at once (intracellular uptake and intra- and extracellular efflux for both single cells and spheroids).

\section{Materials}

Palbociclib (PD 0332991), agarose, phosphate-buffered saline, cell culture reagents, DMSO, and high-performance liquid chromatography grade acetonitrile, water, and formic acid were all purchased from Sigma-Aldrich, UK. For Western blotting, all antibodies were purchased from Cell Signaling Technology, Inc.; all other reagents were purchased from Sigma-Aldrich unless otherwise specified.

\section{Results}

Cell Viability Assay and Western Blot. Cytotoxic activity for palbociclib was not previously reported in vitro; to ensure cell death did not influence uptake and efflux values obtained in our experiments, the cells were first tested for viability. MCF-7 and DLD-1 cell lines were treated at $0.1 \mu \mathrm{M}$ of palbociclib for 480 minutes under the same conditions as those to be used for the uptake and efflux experiments; 98\% and $97 \%$ of cells were viable for MCF-7 and DLD-1, respectively, following incubation with drug.

To account for potential variability between cell lines in uptake/efflux due to binding to the drug target, we tested expression of CDK4 and downstream signaling proteins in the cell lines by performing Western blots of retinoblastoma $(\mathrm{Rb})$, phospho-retinoblastoma ( $\mathrm{pRb}$ ) (serine 780), CDK4, and CDK6. Both cell lines were positive for Rb (110 kDa), pRb (110 kDa), and CDK4 $(30 \mathrm{kDa})$ and negative (i.e., below detectable levels) for CDK6 (37 kDa). The MCF-7 cell line had higher CDK4 expression than the DLD-1 cell line (Fig. 1). A positive blot for CDK6 in HCC38, a breast cancer cell line, is shown in the Supplemental Fig. S4 as a positive control.

Uptake. Uptake of palbociclib into SCS and spheroids over time was measured in MCF-7 and DLD-1 cell lines (Fig. 2; Table 1). In the SCS, the initial rate of change of intracellular concentration was 631.1 and $187.3 \mathrm{nM} \cdot \mathrm{min}^{-1}$ for MCF-7 and DLD-1, respectively. In contrast, in spheroids the initial rate of uptake (log phase) was 31.2 and $15.6 \mathrm{nM} \cdot \mathrm{min}^{-1}$ for the MCF-7 and DLD-1 lines, respectively. In SCS the $C_{\max }$ of intracellular palbociclib was reached at 10 minutes and then plateaued, whereas in spheroids, uptake did not achieve steady state until 120 minutes in DLD- 1 and 240 minutes in MCF-7, respectively. The intracellular palbociclib $C_{\max }$ was far higher than the concentration in the external media, in both cells and spheroids. In MCF-7, the mean $C_{\max }$ was $3.22 \mu \mathrm{M}$ in SCS and $2.91 \mu \mathrm{M}$ in spheroids; this was $32-$ and 29-fold higher, respectively, than the treatment concentration of $0.1 \mu \mathrm{M}$. In the DLD-1 cell line, the mean $C_{\max }$ in SCS was 1.28 , and $0.68 \mu \mathrm{M}$ in spheroids; this is almost 13- and 7-fold higher, respectively, than the external media concentration. The difference in $C_{\max }$ between SCS and spheroids was not, however, statistically significant (Mann-Whitney test $P=$ 0.36 for MCF-7 line and $P=0.2$ for DLD-1 line). Moreover, $C_{\max }$ was higher in the MCF-7 line than in DLD-1 in both SCS

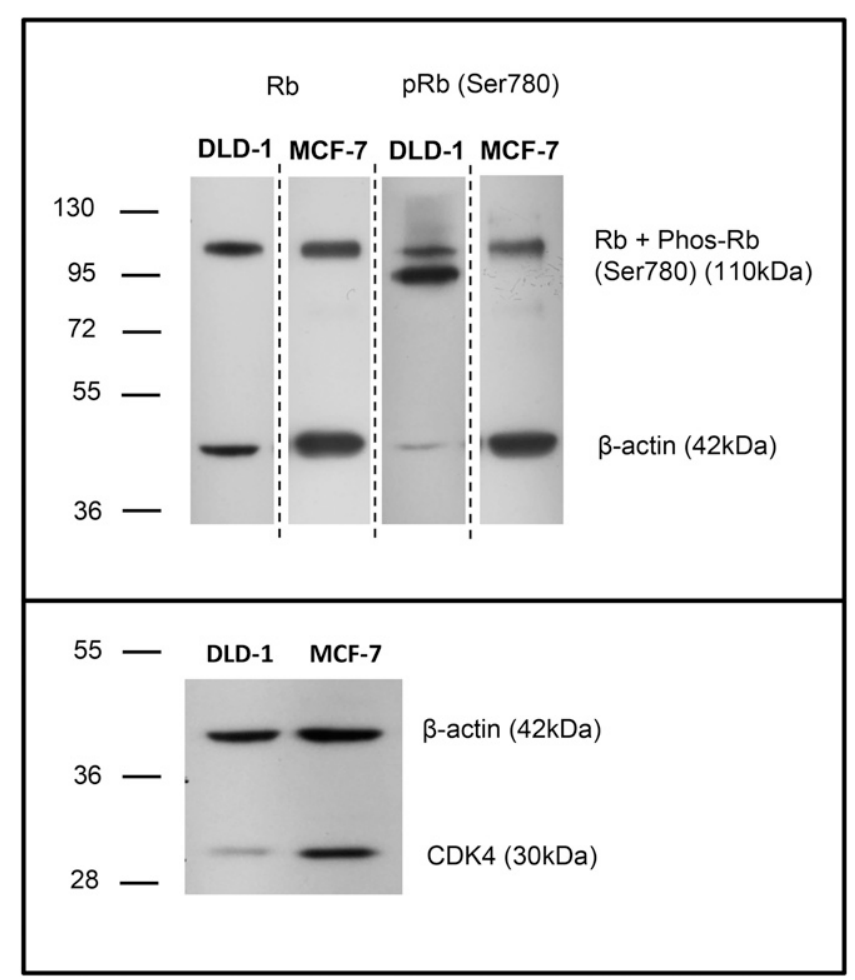

Fig. 1. A representative Western blot of CDK4 (30 kDa), Rb (110 kDa), and pRb phosphorylated at serine $780(110 \mathrm{kDa})$ expression in MCF-7 and DLD-1 cell lines. Negative CDK6 $(37 \mathrm{kDa})$ not shown. 
A

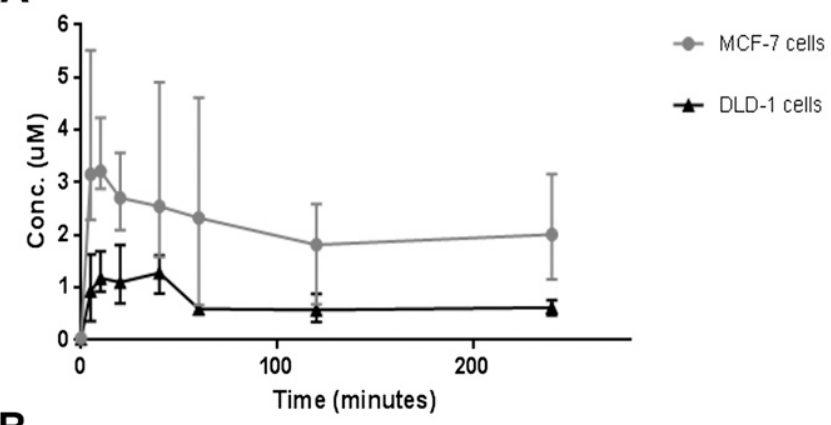

B

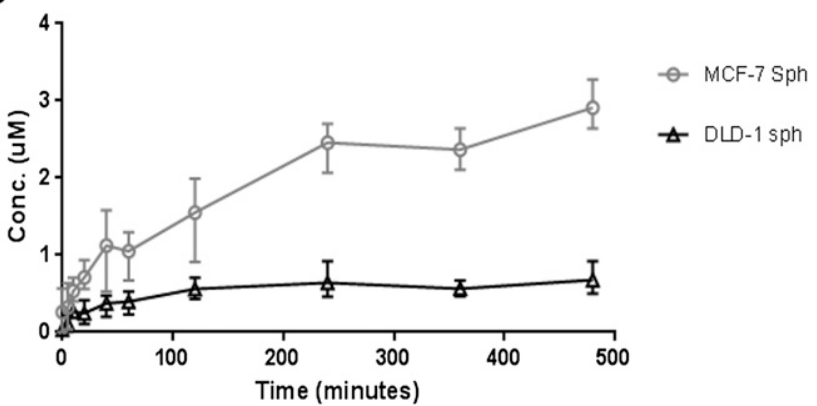

Fig. 2. Palbociclib uptake over time in SCS (A) and spheroids (sph; B) for MCF-7 and DLD-1 cell lines when exposed to media containing $0.1 \mu \mathrm{M}$ palbociclib for up to 480 minutes. Error bars represent range $(n=3)$. Difference in MCF-7 and DLD-1 $C_{\max }$ for SCS and spheroids, MannWhitney test $P=0.035$ and 0.1 , respectively.

and spheroids; the difference was statistically significant only in SCS (Mann-Whitney test: $P=0.035$ for SCS and 0.1 for spheroids).

Total palbociclib uptake, expressed as AUC taken from the drug uptake curve, was lower in DLD-1 [mean $\mathrm{AUC}_{(0-4 \mathrm{~h})} 169$ and $119 \mu \mathrm{M} / \mathrm{h}$ for SCS and spheroids, respectively] than in MCF-7 experiments [mean $\mathrm{AUC}_{(0-4}$ h) 509 and $368 \mu \mathrm{M} / \mathrm{h}$ for SCS and spheroids, respectively]; this difference reached statistical significance in SCS but not the spheroids (MannWhitney test: $P=0.035$ and 0.1 , respectively). These results did not differ significantly when expressed as drug:protein ratio, with a similar pattern of drug uptake reaching $C_{\max }$ at 10 minutes in SCS for both cell lines, and at 120 minutes for DLD-1 and 240 minutes for MCF-7 spheroids (Supplemental Fig. S5)

The initial rate of uptake (log phase) was 10.29 and 2.25 pmol. $\mathrm{mg}^{-1} \cdot \min ^{-1}$ for the MCF-7 and DLD-1 SCS, respectively. In spheroids the initial rate of uptake (log phase) was 0.29 and $0.10 \mathrm{pmol} \cdot \mathrm{mg}^{-1} \cdot \mathrm{min}^{-1}$ for the MCF-7 and DLD-1 lines, respectively. Mean $C_{\max }$ in MCF-7 was $53.5 \mathrm{pmol} \cdot \mathrm{mg}^{-1}$ for SCS and $31.6 \mathrm{pmol} \cdot \mathrm{mg}^{-1}$ for spheroids. DLD-1 mean $C_{\max }$ was 15.29 and $8.11 \mathrm{pmol} \cdot \mathrm{mg}^{-1}$ for cells and spheroids, respectively. Total palbociclib uptake, expressed as mean $\left.\mathrm{AUC}_{(0-4} \mathrm{h}\right)$, for the uptake curve in DLD-1 was 2440 and $1510 \mathrm{pmol} \mathrm{h} . \mathrm{mg}^{-1}$ for SCS and spheroids, respectively, and for MCF-7 was 7535 and 4689 pmol h. $\mathrm{mg}^{-1}$ for SCS and spheroids, respectively. The differences in palbociclib uptake of SCS between DLD-1 and MCF-7 for $C_{\max }$ and AUC did not reach statistical significance when expressed as drug:protein ratio $(P=0.057$ and $P=0.057$, respectively).

Efflux. Efflux of palbociclib from cells and spheroids was measured over time after 1 and 2 hours, respectively, of drug exposure in MCF-7 and DLD-1 cell lines (Fig. 3; Table 2). Drug exposure time prior to efflux experiments was selected based on the time taken to reach $C_{\max }$ in uptake experiments. Exposure time was, therefore, longer in spheroids as drug uptake was slower. This ensured the initial intracellular drug concentration was close to steady state before efflux commenced and limited the potential for experimental error.

The rate of change of intracellular concentration of palbociclib during efflux studies in SCS and spheroids was -2.3 and $-2.1 \mathrm{nM} \cdot \mathrm{min}^{-1}$, respectively, in the MCF-7 cell line. In the DLD-1 cell line, palbociclib efflux was -0.08 and -0.1 $\mathrm{nM} \cdot \mathrm{min}^{-1}$, respectively, in SCS and spheroids. In MCF-7, efflux equilibrium was achieved somewhat later than in the DLD-1. In SCS efflux, equilibrium was reached at 480 minutes in MCF-7 and 240 minute in DLD-1. In spheroid efflux, DLD-1 reached equilibrium at 240-360 minutes, while MCF-7 had not yet reached equilibrium by 480 minutes (the latest time point measured). Again, these results were similar when expressed as drug-to-protein ratio (Supplemental Fig. S6). The ratio of intracellular to extracellular drug was calculated at each time point (Supplemental Fig. S7). In MCF-7, the percentage of intracellular palbociclib remaining at 480 minutes was $45.14 \%$ and $70.76 \%$ in the SCS and spheroids, respectively. In DLD-1, the percentage remaining drug was $30.99 \%$ and $40.67 \%$ in SCS and spheroids, respectively.

Mathematical Model. The results obtained using the mathematical model, shown in Figs. 4 and 5, reproduce qualitatively most of the features in the experimental data and distinguish between SCS and spheroid data. The simplicity of the model precludes it from being able to reproduce the decrease in intracellular drug concentrations from their high early values in the SCS uptake experiments as seen in Figs. 4 and 5. In particular, the high intracellular drug concentrations visible in the first 30-60 minutes of the SCS uptake experiments are not approximated well by the simulations; by contrast, the model recapitulates the observed concentration time variation for the spheroids reasonably well. The results for DLD-1 do show a very rapid initial increase in intracellular

TABLE 1

Summary of uptake data for MCF-7 and DLD-1 in SCS and spheroids

Values in brackets represent respective confidence intervals of 95\% ( $\mathrm{IC}_{95} \%$ ). Difference in SCS and spheroid $C_{\max }$ not significant (MCF-7 $P=0.36$, DLD-1 $\left.P=0.2\right)$. Difference in MCF-7 and DLD-1 $C_{\max }$ SCS $P=0.035$ (significant), spheroids $P=0.1$ (not significant). Difference in DLD-1 and MCF-7 AUC SCS $P=0.035$ (significant), AUC spheroids $P=$ 0.1 (not significant).

\begin{tabular}{lrrrrr}
\hline Uptake & Uptake & $T_{\max }$ & \multicolumn{1}{c}{$C_{\max }$} & \multicolumn{1}{c}{$C_{\max }$} \\
& $n M / \min$ & $\min$ & $\mu M$ & $p m o l / m g$ & \multicolumn{1}{c}{ AUC } \\
MCF-7 SCS & 631.1 & 10 & $3.22 \mu \mathrm{M}(2.50-3.93)$ & $53.5(43.5-63.5)$ & $509(237.7-779.8)$ \\
DLD-1 SCS & 187.3 & 10 & $1.28(0.38-2.19)$ & $15.29(7.92-22.6)$ & $169(29.73-308.3)$ \\
MCF-7 spheroids & 31.2 & 240 & $2.91(2.08-3.73)$ & $31.6(21.8-41.4)$ & $624(428.1-819.3)$ \\
DLD-1 spheroids & 15.6 & 120 & $0.68(0.14-1.22)$ & $8.11(2.30-13.9)$ & $153(66.29-324.3)$ \\
\hline
\end{tabular}


A

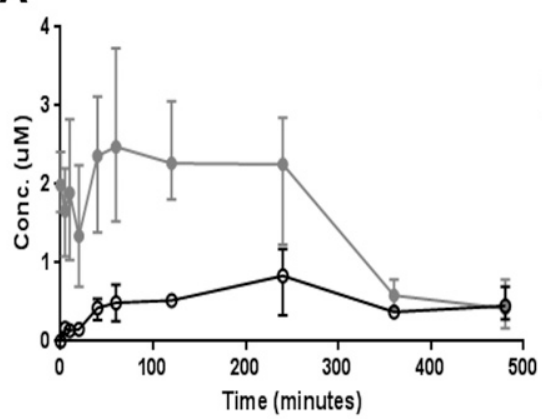

C

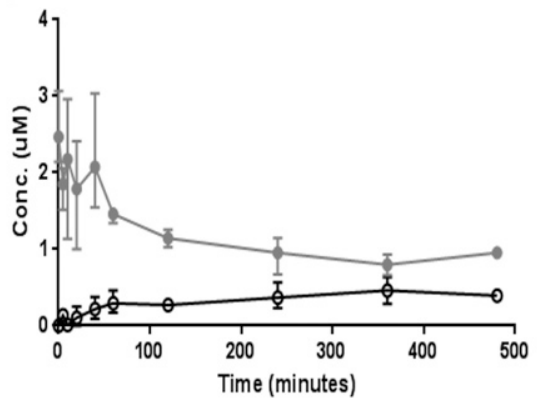

B

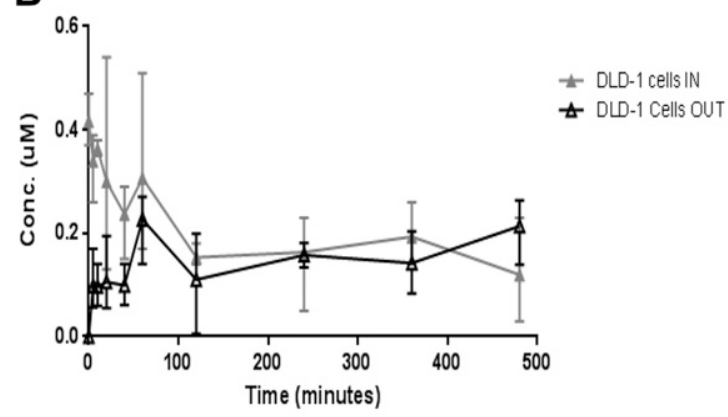

D

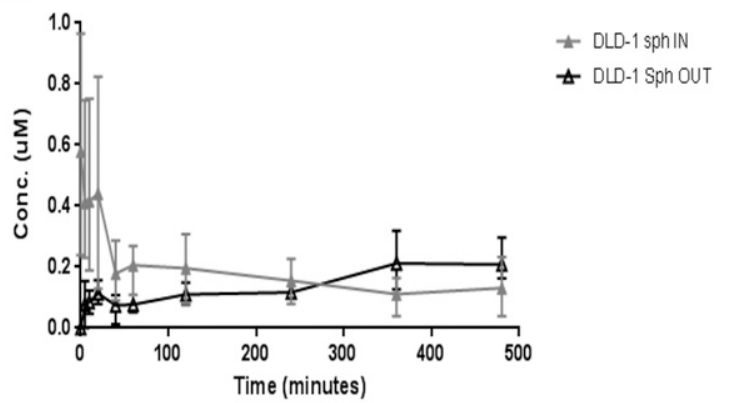

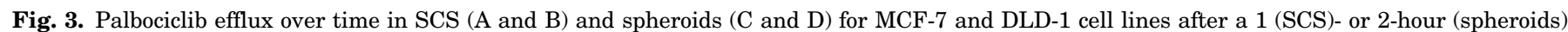

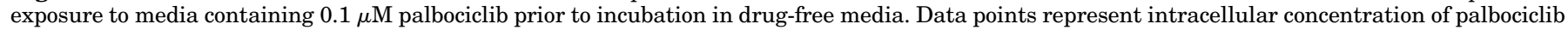
remaining at each time point (IN) and effluxed palbociclib measured in the media $($ OUT). Error bars represent range $(n=3)$.

concentrations, but it is much smaller in magnitude than seen in the experiments. For DLD-1, the simulations slightly overestimated the SCS extracellular efflux concentrations and underestimate the spheroid extracellular efflux concentrations, but otherwise fit well to the experimental data. For MCF-7, the fits also qualitatively match the experimental data, although the intracellular concentrations obtained from the simulations slightly overestimate the uptake data and underestimate the efflux data.

Note that the data have been fitted with both a standard least squares fit (shown in Figs. 4 and 5) and a $\chi^{2}$ fit (Supplemental Annex 1). There were no significant qualitative differences between the two sets of results. The discrepancies between the observed data and model fits for the SCS data are informative; it is clear that the initial very rapid uptake of drug in the SCS is not described accurately by simple firstorder kinetic processes that are assumed in the model and that an active transport mechanism is more likely, with higherorder terms playing a leading role. Conversely, the agreement between the model and experiment seen in the spheroids, arguably of more relevance in tumors in vivo, confirms that the dominant drug transport processes in the spheroid can be reasonably approximated by first-order kinetic/diffusive transport mechanisms.

\section{Discussion}

We developed a methodology to study drug uptake and efflux in vitro in $2 \mathrm{D}$ and $3 \mathrm{D}$ models. For palbociclib, we observed very high intracellular drug concentrations compared with the external media drug treatment concentration, with especially rapid uptake in SCS. The mathematical model we developed was able to reproduce most of the features of the experimental data.

Palbociclib uptake experiments showed an initial rapid uptake rate in SCS, with slower uptake in spheroids occurring over 2 hours. In contrast, palbociclib efflux was much slower. Notably, intracellular $C_{\max }$ in both SCS and spheroids was up to 32 times higher than the external media concentration. There are a number of potential hypotheses to explain this unusual pattern of drug uptake and efflux, such as active uptake or facilitated transport of the drug across the cell

TABLE 2

Summary of efflux data for MCF-7 and DLD-1 in SCS and spheroids Values in brackets represent respective confidence intervals of $95 \%$ ( $\mathrm{IC}_{95} \%$ ).

\begin{tabular}{lccc}
\hline Efflux & Efflux & Time to Efflux Re-equilibration & Percentage of Intracellular Palbociclib Remaining at 480 min \\
\hline & $n M / \min$ & $\min$ & $\%$ \\
MCF-7 cells & 2.3 & 480 & $45.1(26.1-64.2)$ \\
DLD-1 cells & 0.08 & 240 & $31(0-68.54)$ \\
MCF-7 spheroids & 2.1 & $>480$ & $70.76(65.65-75.87)$ \\
DLD-1 spheroids & 0.1 & $240-360$ & $40.67(31.11-50.27)$ \\
\hline
\end{tabular}


A

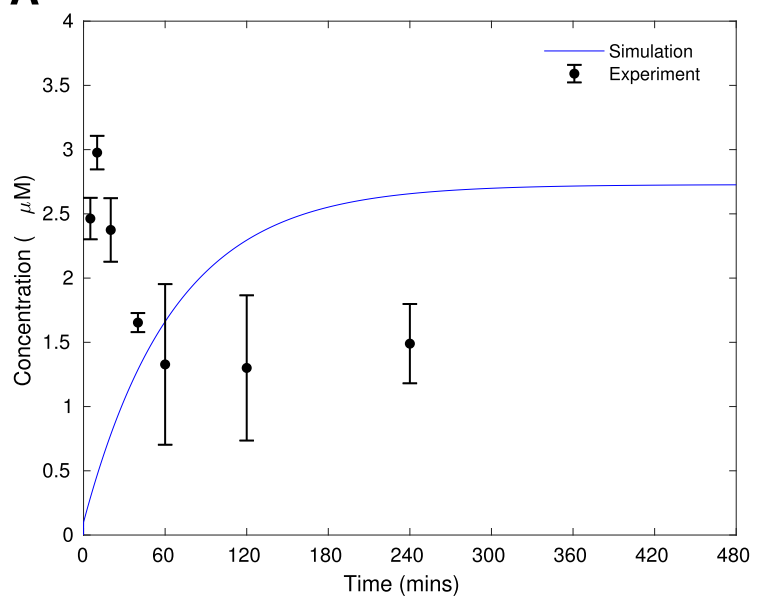

B

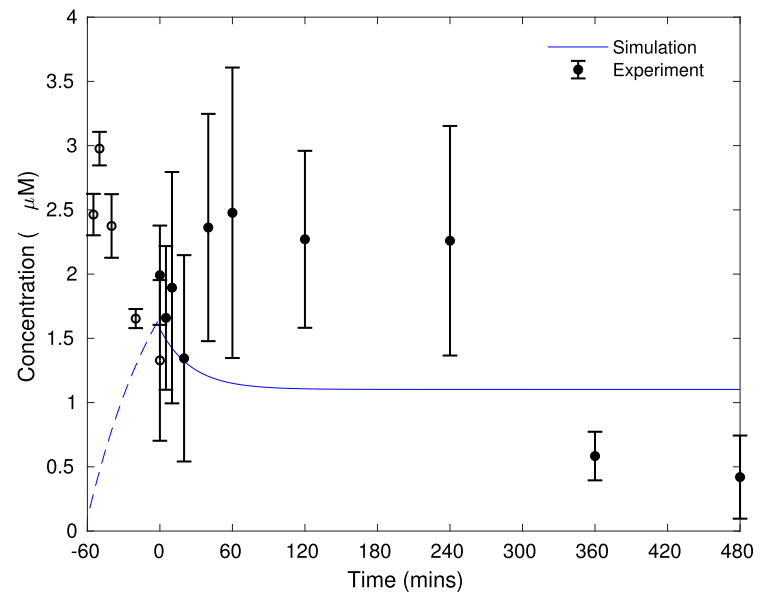

C

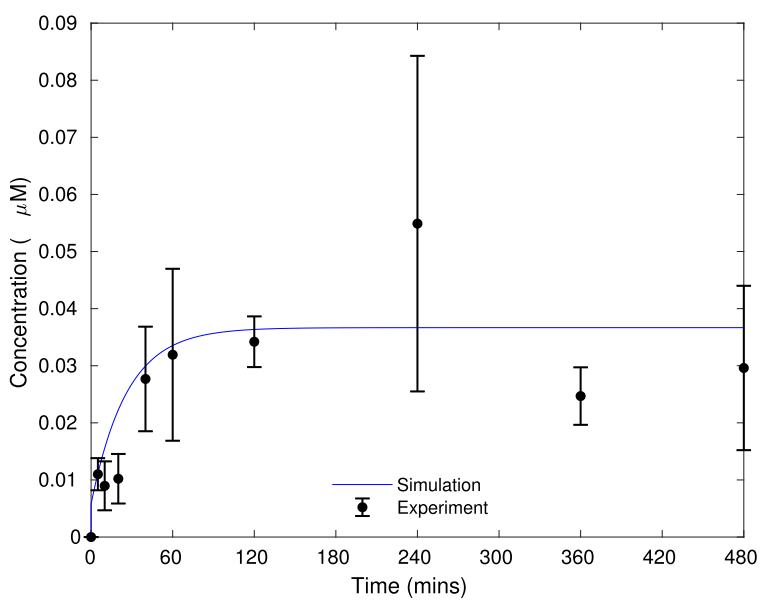

D

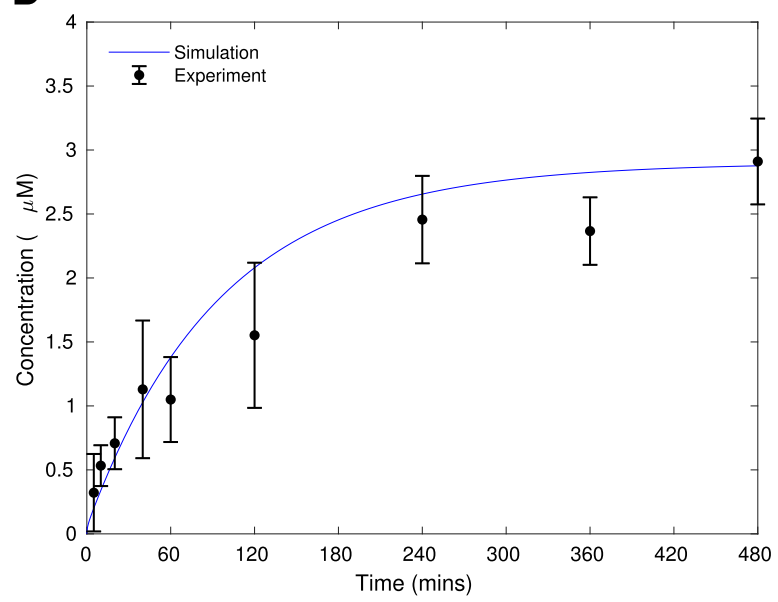

E

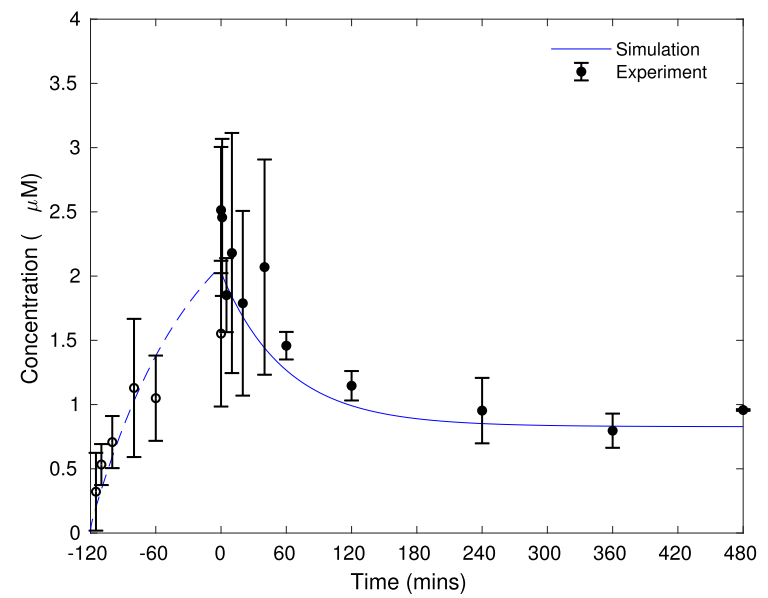

$\mathbf{F}$

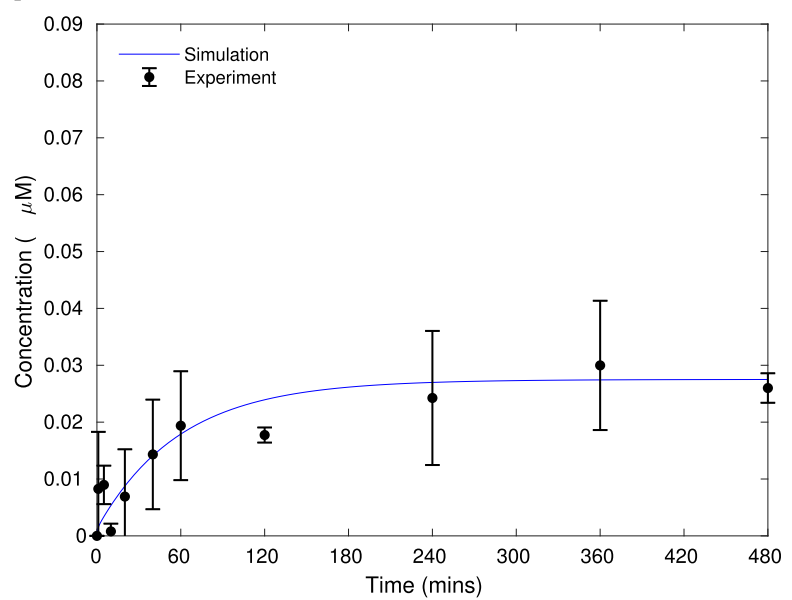

Fig. 4. Least squares five-parameter fitting model to all cell and spheroids data for MCF-7. The graphs represent uptake intracellular concentration for SCS (A) and spheroids (D), efflux intracellular concentration for SCS (B) and spheroids (E) and efflux extracellular concentration measured in the media for SCS (C) and spheroids (F). Data points are experimental data as shown in Figs. 2 and 3. The line on each plot represents the mathematical simulation.

membrane, and very high nontarget specific intracellular protein binding. Indeed, palbociclib has high peripheral tissue and protein binding, evidenced by its very large volume of distribution (www.ema.europa.eu). When palbociclib crosses the cell membrane, it quickly binds intracellular components leaving a low concentration of free, unbound drug. Drug will, therefore, continue to move into the cell until equilibrium is reached between the free intracellular drug concentration and the media, leading to accumulation inside the cells. In spheroids, uptake is much slower as it is likely that intracellular binding saturation must be reached across several layers of cells. The less pronounced differences in efflux 
A

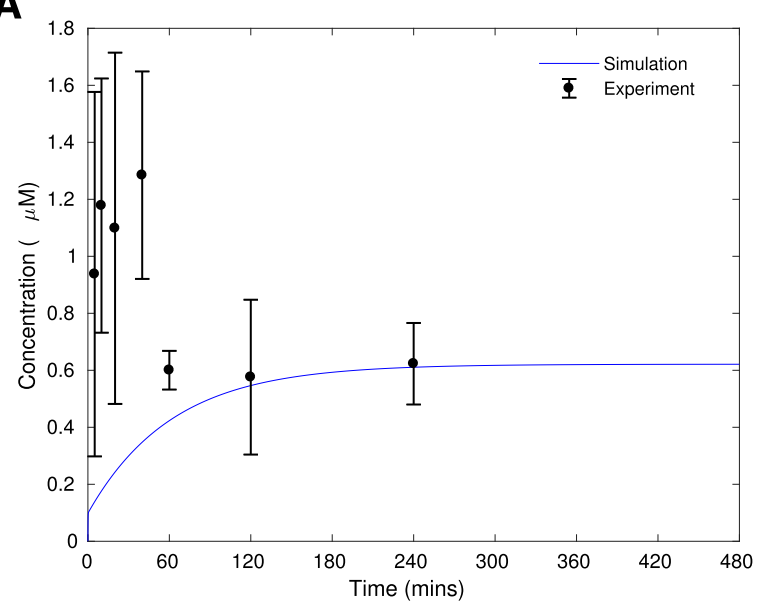

B

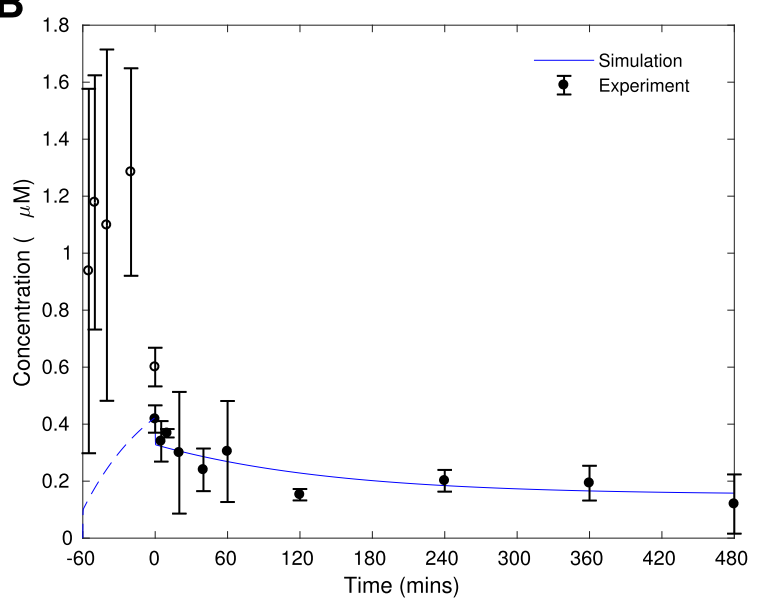

C

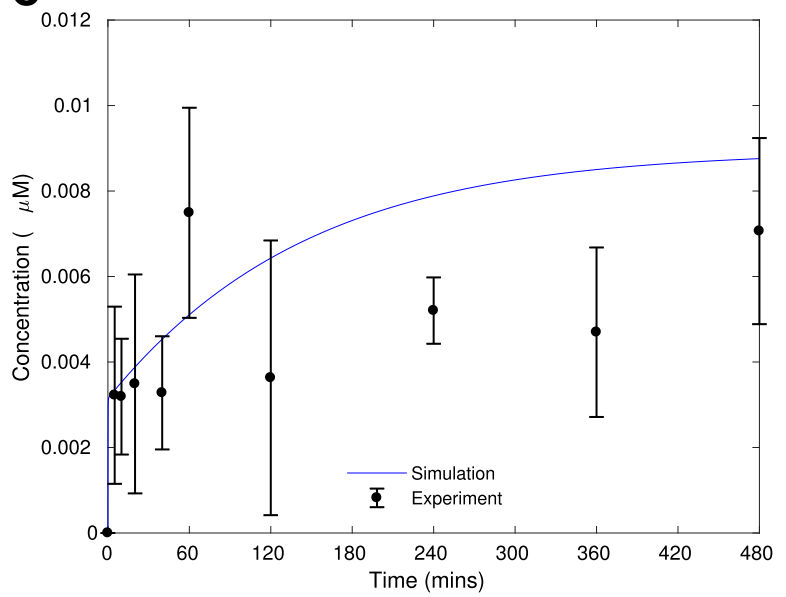

D

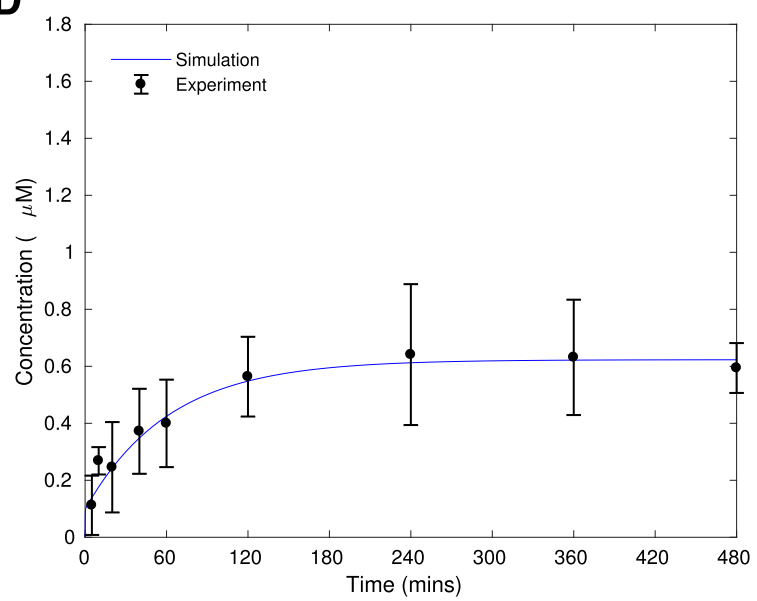

E

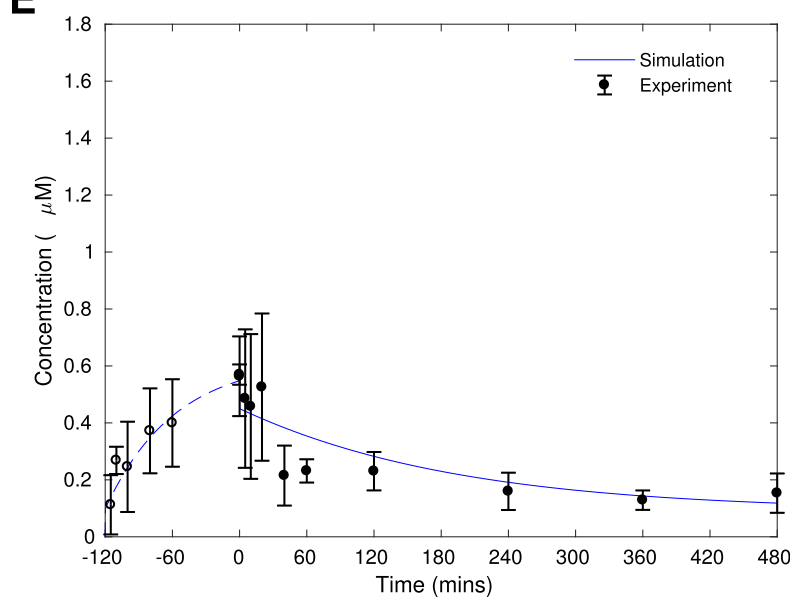

$\mathbf{F}$

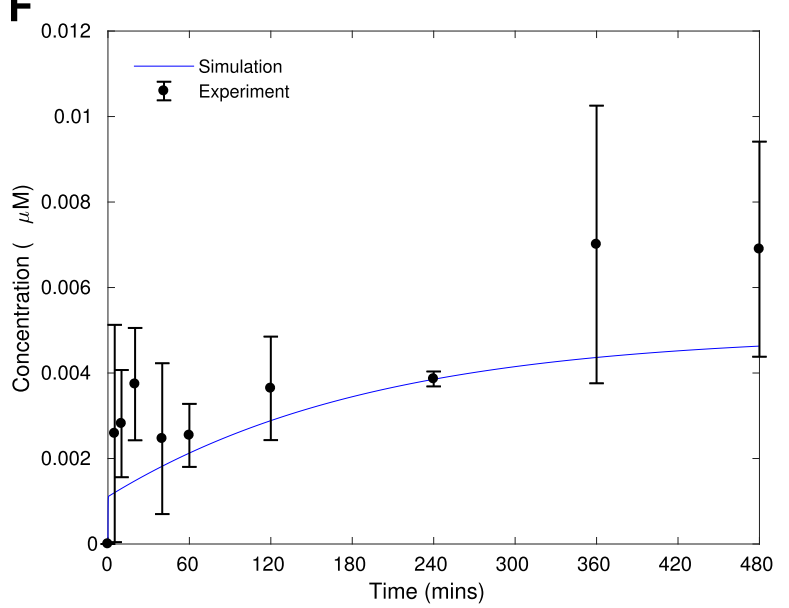

Fig. 5. Least squares five-parameter fitting model to all cell and spheroids data for DLD-1. The graphs represent uptake intracellular concentration for SCS (A) and spheroids (D), efflux intracellular concentration for SCS (B) and spheroids (E) and efflux extracellular concentration measured in the media for SCS (C) and spheroids (F). Data points are experimental data as shown in Figs. 2 and 3. The line on each plot represents the mathematical simulation.

between single cells and spheroids, when compared with uptake, could be explained by a slow intracellular unbinding rate.

There are differences between the data obtained for MCF-7 and DLD-1; the uptake of palbociclib is more prominent in MCF-7, reaching a $C_{\max }$ three times higher than in DLD-1.
Potential differentials in transporter protein expression between the cell lines are the most likely explanation for this observation; indeed, the presence of an efflux transporter may explain why efflux equilibrium is reached more rapidly in DLD-1 cells. Palbociclib is a known substrate of the efflux transporter Pgp and breast cancer resistance protein (De 
Gooijer et al., 2015; Parrish et al., 2015); we have not yet explored the different transporters expressed in these two cell lines but this would benefit from further study.

The experimental data are largely supported by the mathematical modeling. Specifically, the parameter values obtained and the model simulations suggest that binding is more rapid than unbinding. As in the experiments, the intracellular concentrations of bound drug at steady state in the uptake model simulations are much higher than the initial concentration of drug in the extracellular media (27 times higher for MCF-7, 6 times higher for DLD-1). These high concentrations are reached quite rapidly; hence, the binding in our model must be rapid, as higher intracellular concentrations than those in the external media could not be achieved by passive transport across the cell membrane alone and there is no facilitated membrane transport in our simple mathematical model. Moreover, the nature of the efflux data requires at least one process to act over a period of hours; only slow unbinding can give the slow decrease in intracellular efflux concentrations because the initial rapid rise in the extracellular concentrations suggests that membrane transport is rapid. This is more pronounced in the simulations of the DLD1 data, despite the MCF-7 data signifying much faster membrane transport (according to the fitted values of $k_{1}$ ). The slowest process (i.e., unbinding) is faster for MCF-7; therefore, the single-cell simulations settle more rapidly to an equilibrium state, most noticeably in the efflux experiments. However, the ratio of binding to unbinding rates is significantly higher for DLD-1, and consequently the equilibrium is closer to saturation in the uptake experiments with $\mathrm{C}_{3}$, the bound drug concentration, reaching approximately $0.6 \%$ of $\mathrm{C}_{0}$ at steady state for MCF-7 compared with approximately 85\% for DLD-1.

The model for the spheroid data allows for spatial variation of drug concentrations within each spheroid. For the DLD-1 extracellular efflux data, a rapid initial increase suggests that transport within the spheroid is rapid, allowing unbound drug to escape quickly. This is followed by a slower process in which the bound drug starts to leave the spheroid. A consequence of this rapid intraspheroid transport is that the model simulations suggest that the SCS and spheroid drug concentrations should both move toward equilibrium at a similar rate when considering uptake. In contrast, the MCF-7 data suggest much slower transport within the spheroid, so both uptake and efflux occur more slowly in spheroids than in SCS. This may, in part, be due to the binding of drug in the periphery of the spheroids delaying the transport of drug to their centers. In the simulations, this difference is a consequence of a much lower diffusion rate (Supplemental Materials 1; Table 1) computed when the model parameters are fitted to the MCF7 data. When a $\chi^{2}$ fit is used, slower uptake and efflux is again seen in spheroids for MCF-7, but with this parameterization it is a higher binding rate that induces this behavior in combination with relatively slow diffusion. This provides some support to the hypothesis that high protein binding might explain the differences between the single cell and spheroid data, although the model also suggests that slow diffusive transport of the drug within the spheroid would affect the concentration profiles in a similar way.

It is difficult to compare our results with the clinical PK parameters of palbociclib, as intratumoural concentrations of palbociclib have not been reported in this setting. Moreover, our exposure concentration of $0.1 \mu \mathrm{M}$ should be seen as an artificial representation of steady-state extracellular palbociclib concentrations based on reported clinical plasma $C_{\max }$ values. Nevertheless, intratumoural palbociclib concentrations from in vivo experiments with breast cancer xenografts have been reported. There was, however, no correlation of plasma and tumor concentrations, because the mice bearing breast cancer xenografts for each measurement were treated with different palbociclib doses. In preclinical studies, palbociclib intratumoral concentrations have been reported to be $25,163 \mathrm{ng} / \mathrm{g}(56.2 \mu \mathrm{M})$ at 6 hour after an oral dose of $100 \mathrm{mg} / \mathrm{kg}$, and plasma $C_{\max }$ of $48 \mathrm{ng} / \mathrm{ml}(0.11 \mu \mathrm{M})$ after a dose of $2 \mathrm{mg} / \mathrm{kg}$ (Nguyen et al., 2010; Smith et al., 2011). Human plasma $C_{\max }$ from a palbociclib phase I study was $97.4 \mathrm{ng} / \mathrm{ml}(0.21 \mu \mathrm{M})$ in a multiple dose schedule, but again tumor palbociclib levels were not measured (Flaherty et al., 2012). The high volume of distribution in clinical data supports, however, the high intracellular binding of palbociclib.

Similar experiments have been undertaken with $5 \mathrm{FU}$, a drug with contrasting physicochemical properties to palbociclib, being highly polar with low protein and tissue binding. Images of radiolabeled 5FU showed very rapid uptake in spheroids within a few minutes (Nederman and Carlsson, 1984). Our 5FU uptake and efflux experiments (data not shown) are consistent with this previous publication, showing no difference between SCS and spheroids with similar intracellular and extracellular concentrations at $C_{\max }$ and steady state (Jove et al., 2017). In contrast, studies undertaken with doxorubicin, a drug with physicochemical properties more similar to palbociclib, also showed quicker drug uptake in monolayers than in spheroids (Sutherland et al., 1979; Shan et al., 2018).

Our results demonstrate the importance of a drug's physicochemical properties in penetration and distribution within cancer cells. The results obtained using the mathematical model provide additional support for the hypotheses derived from the experimental data regarding the mechanisms that govern the dynamics of palbociclib intratumoural PK. It should be noted that in vitro models do not incorporate other factors typically affecting drug delivery in vivo, such as tumor stroma or vasculature, and our mathematical model omits the effects of active transport, blood supply, and interstitial fluid pressure. The parameterization itself determines how features in the experimental data are interpreted by the model.

Arguably, the most striking data from this study relate to the large intracellular concentrations that accumulate rapidly after drug exposure. As drugs are assessed in vitro for their cytotoxicity or inhibitory effect, as expressed by an $\mathrm{IC}_{50}$, the implications of the intracellular concentrating effect we have seen could be important, with potential consequences for the translation of $\mathrm{IC}_{50}$ values into clinical efficacy. If intracellular concentrations in vitro are significantly higher than treatment concentration, actual $\mathrm{IC}_{50}$ values for some drugs may be much higher than previously thought. This may potentially lead to treatment failures due to suboptimal dosing. We anticipate that, although some improvements are warranted as more data become available, the mathematical model developed here may be used to gain insights into the mechanisms underpinning the data and to obtain accurate predictions of the movement of drugs through tissue based on plasma concentrations. It is also anticipated that this will assist extrapolation from plasma PK data to tumor concentrations, 
estimating tumor PK data without the need for repeat tumor biopsy.

In conclusion, these are the first in vitro models of drug uptake and efflux for palbociclib, and, together with the mathematical model, they are useful to understand palbociclib intratumoral distribution. They could potentially be applied to other drugs to elucidate their intratumoural PK, facilitating improvements in drug delivery and ultimately treatment efficacy.

\section{Acknowledgments}

Special acknowledgment to Spanish Medical Oncology Society (SEOM) for contribution with a personal grant for research abroad of Dr. M. Jove and Dr. Ramon Salazar for his contribution in this paper.

\section{Authorship Contributions}

Participated in research design: Jove, Spencer, Loadman, Twelves. Conducted experiments: Jove, Spencer.

Contributed analytical tools and performed data analysis: Hubbard, Holden, O'Dea, Brook, Smye.

Wrote or contributed to the writing of the manuscript: Jove, Spencer, Hubbard, Holden, O'Dea, Brook, Phillips, Smye, Loadman, Twelves.

\section{References}

Altrock PM, Liu LL, and Michor F (2015) The mathematics of cancer: integrating quantitatve models. Nat Rev Cancer 15:730-745.

Babuška I (1976) Homogenization approach in engineering, pp 137-153, Springer Berlin Heidelberg, Berlin, Heidelberg.

Barrera-Rodríguez R and Fuentes JM (2015) Multidrug resistance characterization in multicellular tumour spheroids from two human lung cancer cell lines. Cancer Cell Int 15:47.

Clark AS, Karasic TB, DeMichele A, Vaughn DJ, O'Hara M, Perini R, Zhang P, Lal P Feldman M, Gallagher M, et al. (2016) Palbociclib (pd0332991)-a selective and potent cyclin-dependent kinase inhibitor: a review of pharmacodynamics and clinical development. JAMA Oncol 2:253-260

Dalwadi M, Wang Y, King J, and Minton N (2018) Upscaling diffusion through firstorder volumetric sinks: a homogenization of bacterial nutrient uptake. SIAM J Appl Math 78:1300-1329.

Dean JL, McClendon AK, and Knudsen ES (2012) Modification of the DNA damage response by therapeutic CDK4/6 inhibition. J Biol Chem 287:29075-29087.

de Gooijer MC, Zhang P, Thota N, Mayayo-Peralta I, Buil LCM, Beijnen JH, and van Tellingen $\mathrm{O}(2015) \mathrm{P}$-glycoprotein and breast cancer resistance protein restrict the brain penetration of the CDK4/6 inhibitor palbociclib. Invest New Drugs 33 1012-1019.

Evans CJ, Phillips RM, Jones PF, Loadman PM, Sleeman BD, Twelves CJ, and Smye SW (2009) A mathematical model of doxorubicin penetration through multicellular layers. $J$ Theor Biol 257:598-608.

Flaherty KT, Lorusso PM, Demichele A, Abramson VG, Courtney R, Randolph SS, Shaik MN, Wilner KD, O'Dwyer PJ, and Schwartz GK (2012) Phase I, doseescalation trial of the oral cyclin-dependent kinase 4/6 inhibitor PD 0332991, administered using a 21-day schedule in patients with advanced cancer. Clin Cancer Res 18:568-576.

Fuso Nerini I, Morosi L, Zucchetti M, Ballerini A, Giavazzi R, and D'Incalci M (2014) Intratumor heterogeneity and its impact on drug distribution and sensitivity. Clin Pharmacol Ther 96:224-238.

Godugu C, Patel AR, Desai U, Andey T, Sams A, and Singh M (2013) AlgiMatrix ${ }^{\mathrm{TM}}$ based 3D cell culture system as an in-vitro tumor model for anticancer studies. PLoS One 8:e53708.

Groh CM, Hubbard ME, Jones PF, Loadman PM, Periasamy N, Sleeman BD, Smye SW, Twelves CJ, and Phillips RM (2014) Mathematical and computational models of drug transport in tumours. $J R$ Soc Interface 11:20131173.

Holden E (2018) New Effective Descriptions Of Deformable, Adaptively Remodelling Biological Tissue, University of Nottingham, UK.
Huang B-W and Gao J-Q (2018) Application of 3D cultured multicellular spheroid tumor models in tumor-targeted drug delivery system research. $J$ Control Release 270:246-259.

Hubbard ME, Jove M, Loadman PM, Phillips RM, Twelves CJ, and Smye SW (2017) Drug delivery in a tumour cord model: a computational simulation. $R$ Soc Open Sci 4:170014.

Jove M, Loadman P, Spencer J, Sulayman L, Wicks J, Race A, and Twelves C (2017) Intracellular pharmacokinetics of 5FU and palbociclib: Uptake and efflux in disaggregated cells and 3D models. Cancer Research 77 (13 Supplement):4205-4205

Katt ME, Placone AL, Wong AD, Xu ZS, and Searson PC (2016) In vitro tumor models: advantages, disadvantages, variables, and selecting the right platform. Front Bioeng Biotechnol 4:12

Larsen EW (1975) Neutron transport and diffusion in inhomogeneous media. I. J Math Phys 16:1421-1427.

Masuda N, Nishimura R, Takahashi M, Inoue K, Ohno S, Iwata H, Mori Y, Hashigaki S, Muramatsu Y, Nagasawa T, et al. (2018) Palbociclib in combination with letrozole as first-line treatment for advanced breast cancer: a Japanese phase II study. Cancer Sci 109:803-813.

McClendon AK, Dean JL, Rivadeneira DB, Yu JE, Reed CA, Gao E, Farber JL, Force T, Koch WJ, and Knudsen ES (2012) CDK4/6 inhibition antagonizes the cytotoxic response to anthracycline therapy. Cell Cycle 11:2747-2755.

Minchinton AI and Tannock IF (2006) Drug penetration in solid tumours. Nat Rev Cancer 6:583-592.

Nederman T and Carlsson $\mathrm{J}$ (1984) Penetration and binding of vinblastine and 5fluorouracil in cellular spheroids. Cancer Chemother Pharmacol 13:131-135.

Nguyen L, Zhong W-Z, Painter CL, Zhang C, Rahavendran SV, and Shen Z (2010 Quantitative analysis of PD 0332991 in xenograft mouse tumor tissue by a 96-well supported liquid extraction format and liquid chromatography/mass spectrometry. $J$ Pharm Biomed Anal 53:228-234.

Parrish KE, Pokorny J, Mittapalli RK, Bakken K, Sarkaria JN, and Elmquist WF (2015) Efflux transporters at the blood-brain barrier limit delivery and efficacy of cyclin-dependent kinase 4/6 inhibitor palbociclib (PD-0332991) in an orthotopic brain tumor model. $J$ Pharmacol Exp Ther 355:264-271.

Sanchez-Palencia E (1970) Solutions periodique par rapport aux variables d'espace et applications. C R Acad Sci Paris Ser A 271:1129-1132.

Schüller J, Cassidy J, Dumont E, Roos B, Durston S, Banken L, Utoh M, Mori K, Weidekamm E, and Reigner B (2000) Preferential activation of capecitabine in tumor following oral administration to colorectal cancer patients. Cancer Chemother Pharmacol 45:291-297.

Schwartz GK, LoRusso PM, Dickson MA, Randolph SS, Shaik MN, Wilner KD, Courtney R, and O'Dwyer PJ (2011) Phase I study of PD 0332991, a cyclindependent kinase inhibitor, administered in 3-week cycles (Schedule 2/1). Br $J$ Cancer 104:1862-1868.

Shan F, Close DA, Camarco DP, and Johnston PA (2018) High-content screening comparison of cancer drug accumulation and distribution in two-dimensional and three-dimensional culture models of head and neck cancer. Assay Drug Dev Technol 16:27-50.

Shipley RJ and Chapman SJ (2010) Multiscale modelling of fluid and drug transport in vascular tumours. Bull Math Biol 72:1464-1491.

Smith D, Tella M, Rahavendran SV, and Shen Z (2011) Quantitative analysis of PD 0332991 in mouse plasma using automated micro-sample processing and microbore liquid chromatography coupled with tandem mass spectrometry. $J$ Chromatogr B Analyt Technol Biomed Life Sci 879:2860-2865.

Sutherland RM, Eddy HA, Bareham B, Reich K, and Vanantwerp D (1979) Resistance to adriamycin in multicellular spheroids. Int J Radiat Oncol Biol Phys $\mathbf{5}$ : $1225-1230$

Tamura K, Mukai H, Naito Y, Yonemori K, Kodaira M, Tanabe Y, Yamamoto N, Osera S, Sasaki M, Mori Y, et al. (2016) Phase I study of palbociclib, a cyclindependent kinase 4/6 inhibitor, in Japanese patients. Cancer Sci 107:755-763.

Taylor JW, Parikh M, Phillips JJ, James CD, Molinaro AM, Butowski NA, Clarke JL Oberheim-Bush NA, Chang SM, Berger MS, et al. (2018) Phase-2 trial of palbociclib in adult patients with recurrent RB1-positive glioblastoma. J Neurooncol 140: 477-483.

Winter U, Aschero R, Fuentes F, Buontempo F, Zugbi S, Sgroi M, Sampor C, Abramson DH, Carcaboso AM, and Schaiquevich P (2019) Tridimensional retinoblastoma cultures as vitreous seeds models for live-cell imaging of chemotherapy penetration. Int J Mol Sci 20:E1077.

IBRANCE® (palbociclib). (2015) FULL PRESCRIBING INFORMATION, Pfizer (Labs P ed). U.S. Food and Drug Administration.

Address correspondence to: P.M. Loadman, Institute of Cancer Therapeutics, University of Bradford, Bradford BD7 1DP, UK. E-mail: p.m.loadman@ bradford.ac.uk; or M. Jove, Institut Català d'Oncologia, Medical Oncology department, Av. Gran Via de L'Hospitalet 199-203, 08908 L'Hospitalet de Llobregat, Barcelona, Spain. E-mail: mjove@iconcologia.net 